\begin{tabular}{|c|c|c|}
\hline $\begin{array}{l}\text { OPEN } \\
\text { JOURNAL } \\
\text { SYSTEMS } \\
\end{array}$ & $\begin{array}{c}\text { Available on line at Directory of Open Access Journals } \\
\text { Journal of Hyperspectral Remote Sensing v.9, n.1 (2019) 21-29 } \\
\text { www.periodicos.ufpe.br/revistas/jhrs }\end{array}$ & $\begin{array}{c}\text { Journal of } \\
\text { Hyperspectral } \\
\text { Remote Sensing } \\
\end{array}$ \\
\hline $\begin{array}{l}\text { ISSN:2237-2202 } \\
\end{array}$ & & wwww.ufpe.br/jhrs \\
\hline
\end{tabular}

\author{
Laízy de S. Azevedo*, Ana Lúcia B. Candeias** \\ * Graduação em Engenharia Cartográfica; Universidade Federal de Pernambuco (UFPE), Recife-PE. E-mail: \\ laizy_azevedo@hotmail.com \\ ** Professor Associado III; Universidade Federal de Pernambuco (UFPE), Recife-PE. E-mail: analucia@ufpe.br
}

Received 28 November 2018; accepted 3 April 2019

\begin{abstract}
Image fusion can be defined as a method that combines images of different spectral and spatial characteristics to synthesize a new image with better spatial resolution than the original multispectral image. This paper shows the results of the quantitative and qualitative analysis of images fused by the IHS technique (intensity, hue and saturation), The area of study was the surroundings of the Itaparica reservoir, Pernambuco. The technique was applied to Landsat8 (Land Remote Sensing Satellite) images, where the OLI sensor bands 2,3,4,5 and 6 were used in the different combinations of RGB and fused by IHS with the panchromatic band to produce images with Better spatial resolution. The spatial resolution of Landsat 8 is 30 meters for the multispectral image and 15 meters for the panchromatic image. After processing, evaluations were made from visual observations and statistical measures such as: mean, variance, correlation and IQI (image quality index). It was possible to perceive visually the gain in the quality of the images, that is, a greater level of detail due to the increase of the spatial resolution. Statistical analysis showed that the best result was for bands 2,3 and 4 .
\end{abstract}

Keywords: image processing, image fusion, IHS-transformation.

\title{
Análise quantitativa e qualitativa da técnica de fusão IHS aplicada a imagens do satélite Landsat8
}

\section{Resumo}

A fusão de imagens pode ser definida como um método que combina imagens de diferentes características espectrais e espaciais para sintetizar uma nova imagem com melhor resolução espacial que a imagem multiespectral original. Este trabalho apresenta os resultados da análise quantitativa e qualitativa de imagens fusionadas pela técnica IHS (intensidade, matiz e saturação), sendo a área de estudo o entorno do reservatório de Itaparica, Pernambuco. A técnica foi aplicada em imagens do Landsat8 (Land Remote Sensing Satellite), em que as bandas 2,3,4,5 e 6 do sensor OLI foram usadas nas diferentes combinações de RGB e fusionadas por IHS com a faixa pancromática para produzir imagens com melhor resolução espacial. A resolução espacial do Landsat 8 é de 30 metros para a imagem multiespectral e 15 metros para a imagem pancromática. Após o processamento, foram feitas avaliações a partir de observações visuais e medidas estatísticas tais como: média, variância, correlação e IQI (índice de qualidade da imagem). Foi possível perceber visualmente o ganho na qualidade das imagens, ou seja, um maior nível de detalhes devido ao aumento da resolução espacial. Através de análises estatísticas observou-se que o melhor resultado foi para as bandas 2,3 e 4.

Palavras-chave: processamento de imagem, fusão de imagem, transformação-HIS.

\section{Introdução}

O sensoriamento remoto vem sendo utilizado como importante ferramenta em pesquisas científicas e monitoramento dos fenômenos ambientais (Magalhães et al., 2011).

Em grande parte desses estudos é preciso que o objeto estudado apresente uma melhor nitidez e precisão. Sendo assim, muitas vezes é imprescindível que as imagens de satélite adquiridas possuam uma boa resolução espacial, espectral e temporal.

No passado, determinadas análises feitas através de satélites como o CBERS e o Landsat, eram limitadas devido a sua média resolução espacial. Já as imagens de alta resolução como o 
Quickbird e Ikonos, apesar da boa qualidade apresentavam a limitação do alto custo de seus produtos (Wang et al., 2017).

Com o passar dos anos, devido aos avanços tecnológicos e estudos voltados para a área de sensoriamento remoto e processamento de imagens, a técnica de fusão de imagens começou a ser difundida e aplicada.

Para Schneider (2001), a fusão de imagens de satélite de diferentes resoluções espaciais tem por objetivo obter uma imagem aprimorada, ou seja, com maior definição dos detalhes espaciais, o que possibilita uma melhor discriminação dos alvos da superfície terrestre.

O processo de fusão de imagens multiespectrais de baixa resolução com imagens pancromáticas com melhor resolução, tem se tornado uma importante ferramenta para melhorar a qualidade das imagens orbitais e, consequentemente, o seu custo/benefício para diversas aplicações. Para isso, são utilizadas diversas técnicas, dentre elas a IHS.

Uma das formas de descrever a cor por meio da percepção fisiológica humana, é através de três atributos: intensidade, matiz e saturação. $\mathrm{O}$ espaço de cores conhecido por IHS (Intensity, Hue, Saturation) é uma forma alternativa ao espaço RGB (Vermelho, Verde, Azul) de representação das cores.

Meneses e Almeida (2012), afirmam que o sistema IHS não é uma teoria de cor e sim uma transformação numérica do espaço RGB para o espaço IHS. Neste espaço de cores, é possível manipular cada componente separadamente, obtendo assim, maior controle sobre o realce de cor, através de deslocamento e operações lineares e não lineares sobre os componentes I, H e S.

A representação gráfica deste espaço é feita por meio de um cone, em que os três atributos básicos: Intensidade (Intensity-I), Saturação (Saturation-S) e Matiz (Hue-H) são representados pela distância de um ponto até a origem do cone, distância radial do ponto até o eixo central do cone e distância radial ao redor dos círculos de saturação e do eixo de intensidade, respectivamente.

A intensidade corresponde a medida do comprimento de onda médio da luz que o objeto reflete ou emite, ou seja, é a quantidade de brilho de uma cena. A saturação está relacionada com o grau de diluição de uma cor pura por uma luz branca. Já a Matiz, é responsável por determinar a cor do objeto, ou seja, define a cor espectral dominante.

O uso dessa técnica para descrever as cores de uma imagem decompondo-as nos componentes de
Intensidade (I), Matiz (H) e Saturação (S), vai além da produção de composições coloridas. Em suma, a transformação IHS pode ser entendida como uma poderosa ferramenta utilizada como uma forma de combinar imagens de diferentes sensores, com diferentes resoluções espaciais. Essa técnica ficou conhecida como fusão de imagens e ela é amplamente usada em várias aplicações de sensoriamento remoto. Seu objetivo principal é aumentar a resolução espacial das imagens multiespectrais de baixa resolução espacial, por meio da utilização de uma imagem de alta resolução espacial, como exemplo, a banda pancromática ou uma imagem de radar.

Por meio da fusão, é possível processar conjuntamente as imagens multiespectrais e a banda pancromática, combinando suas resoluções e obtendo como resultado final uma imagem com alta resolução espectral e espacial.

Neste trabalho, utilizou-se, uma imagem pancromática com resolução espacial de 15 metros e imagens multiespectrais de menor resolução espacial, 30 metros, ambas do satélite Landsat8-OLI.

A série Landsat teve início na segunda metade da década de 60 por meio de um projeto desenvolvido pela Agência Espacial Americana e dedicada exclusivamente à observação dos recursos naturais terrestres. Em 2013, foi lançado o Landsat 8 que é um grande clássico no setor de Observação da Terra e permite gerar imagens de $15 \mathrm{~m}$ coloridas por fusão digital.

Imagens desse satélite podem ser encontradas gratuitamente em bancos de dados como o do Instituto Nacional de Pesquisas Espaciais (INPE) e Serviço Geológico dos Estados Unidos (USGS) o que permite a viabilidade de pesquisas com essas imagens devido ao baixo custo.

As imagens originais e as fusionadas foram comparadas quantitativamente e qualitativamente, com o objetivo de analisar o desempenho da técnica de fusão IHS. Para isso, foram feitas análises visuais e estatísticas como coeficiente de correlação e índice de qualidade da Imagem (IQI).

\section{Materiais e Métodos}

\section{Caracterização da área de estudo}

A área escolhida para a realização deste estudo compreende ao Reservatório de Itaparica que possui $150 \mathrm{~km}$ de extensão e seu entorno.

Localizado na região do Submédio da Bacia do Rio São Francisco e está a $50 \mathrm{Km}$ dos reservatórios Paulo Afonso-Moxotó. Situa-se entre 
os municípios de Petrolândia, Tacaratu, Floresta, Itacuruba e Belém do São Francisco, no Estado de Pernambuco, e os municípios de Glória, Rodelas e Chorrochó, no Estado da Bahia com função principal de geração de energia elétrica (figura 1).

Começou a ser construído em 1979 pela Companhia Hidroelétrica do Vale do São Francisco
(CHESF), sendo finalizada no ano de 1985.

Estima-se, segundo Silva et al. (2007), que em torno de 33.000 hectares ficaram coberto por água depois do enchimento do Reservatório. Dos quais $28.000 \quad(85 \%)$ localizados no estado de Pernambuco e cerca de 5.000 (15\%) no estado da Bahia.

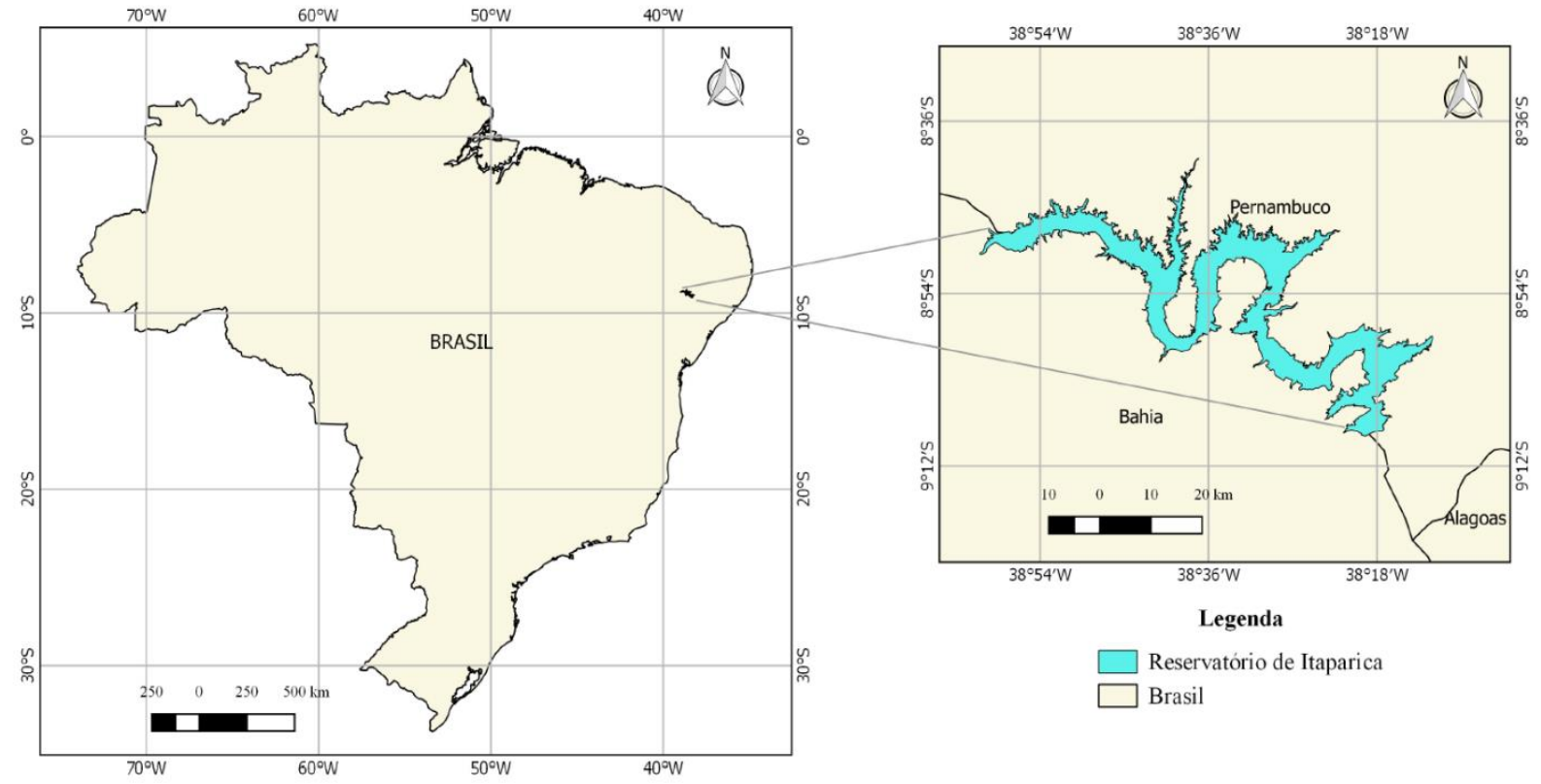

Figura 1 - Localização da área de estudo: Reservatório de Itaparica. Fonte: os autores (2018).

\section{Fusão IHS}

Neste trabalho analisou-se a técnica de fusão de imagens IHS. Como ela trabalha apenas com três bandas por vez, foi feita a fusão das bandas 2,3 e 4 e das bandas 4,5 e 6 com a banda 8 (pancromática). Os experimentos foram realizados utilizando a imagem pancromática e multiespectral adquiridas pelo satélite Landsat8, com características conforme apresentado na Tabela 1 , data de 07 de novembro de 2016, órbita 216 e ponto 066, através do banco de dados do Instituto Nacional de Pesquisas Espaciais (INPE) Todos os testes foram realizados no programa Spring 5.2.7. A análise das medidas estatísticas também foi feita por meio deste programa através da ferramenta análise estatística.

Tabela 1 - Características de algumas bandas espectrais do satélite Landsat8- OLI

\begin{tabular}{ccc}
\hline Bandas espectrais & Faixa Espectral $(\boldsymbol{\mu m})$ & Resolução Espacial $(\mathbf{m})$ \\
\hline Banda2 & $0,45-0,51$ & 30 \\
Banda3 & $0,53-0,59$ & 30 \\
Banda 4 & $0,64-0,69$ & 30 \\
Banda 5 & $0,85-0,88$ & 30 \\
Banda 6 & $1,57-1,65$ & 30 \\
PAN & $0,5-0,68$ & 15 \\
\hline
\end{tabular}

Na Figura 2 é apresentada a composição colorida da imagem multiespectral da área de estudo, com 30 metros de resolução e a imagem pancromática, resolução espacial de 15 metros. 

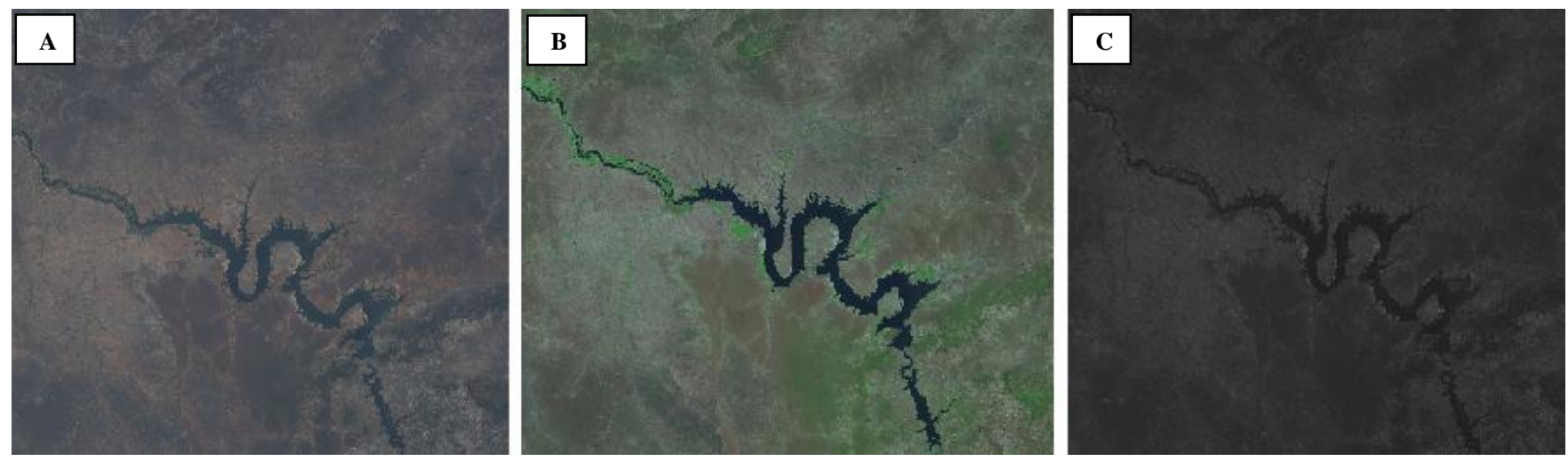

Figura 2 - a) composição colorida banda 2 (B), banda $3(\mathrm{G})$ e banda $4(\mathrm{R})$; b) composição colorida banda 4 (B), banda $5(\mathrm{G})$ e banda $6(\mathrm{R})$; c) Imagem pancromática banda 8.Fonte: os autores (2018).

O procedimento utilizado na fusão de imagens por IHS foi feito basicamente em duas etapas. Na primeira, foram selecionadas três bandas do módulo multiespectral, bandas 2,3,4 e posteriormente 4,5,6, e em seguida realizou-se a transformação do espaço RGB para o espaço IHS, obtendo-se separadamente os componentes de intensidade (I), matiz (H) e saturação (S). Os componentes $\mathrm{H}$ e $\mathrm{S}$ que guardam a informação espectral das cores foram preservados e o componente I descartado e substituído pela imagem pancromática de alta resolução espacial. Na segunda etapa, foi feita a reamostragem dos componentes $\mathrm{H} \mathrm{e}$ $S$ para a resolução espacial da banda pancromática, sintetizando uma imagem colorida com resolução espacial da banda pancromática e com as cores do módulo multiespectral. Assim, foi realizada a transformação inversa para o espaço de cor RGBFigura 3.

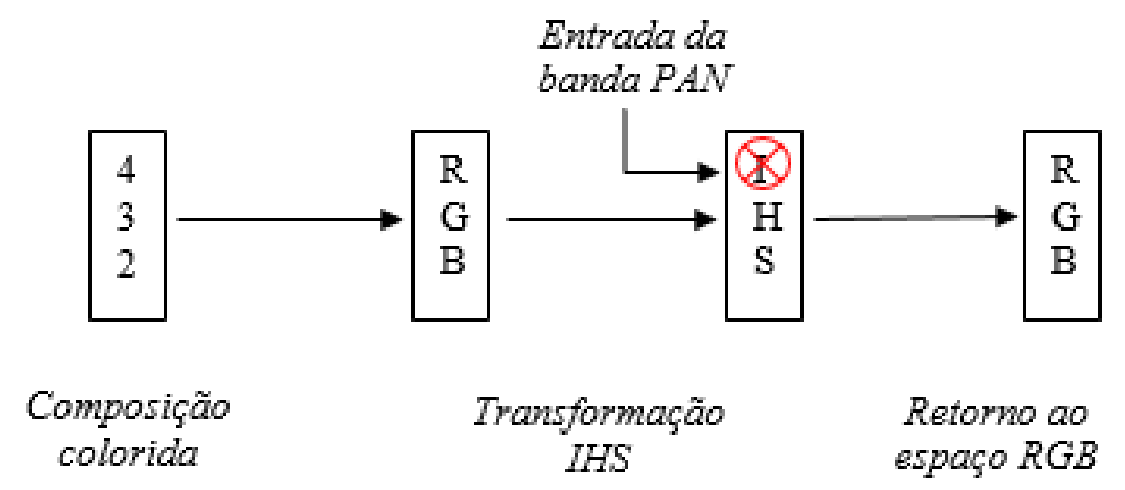

Figura 3 - Etapas do processo de transformação do espaço RGB para IHS. Fonte: Adaptado Meneses e Almeida (2012)

\section{Testes para avaliação da técnica}

Como as imagens não possuem a mesma resolução espacial, neste caso, não existe a possibilidade de se comparar a imagem fusionada à imagem original. Sendo assim, antes do processo de fusão, as imagens Landsat 8 (pancromática e multiespectral) tiveram de ser degradadas, de tal forma que as imagens fusionadas tivessem a mesma resolução espacial da imagem multiespectral original. A fusão entre as imagens degradadas é considerada uma fusão simulada somente para efeito de avaliação. Desta forma, foram geradas, com o interpolador vizinho mais próximo, uma imagem pancromática simulada com resolução de $30 \mathrm{~m}$ e uma multiespectral de $45 \mathrm{~m}$ de resolução.

A fusão entre essas duas imagens gerou uma nova imagem fusionada com resolução de $30 \mathrm{~m}$.

Pushparaj e Hegde (2017) afirmam que existem diversos indicadores visuais e estatísticos para avaliar a qualidade das imagens fusionadas. No 
entanto, não existe um padrão definido de medidas de qualidade em processamento digital de imagens.

Sendo assim, as imagens foram avaliadas qualitativamente e quantitativamente.

$\mathrm{Na}$ avaliação visual foi verificado o resultado geral da fusão em relação à preservação das bordas, cores e do espectro da imagem de alta resolução em relação à imagem de baixa resolução. Porém, esse processo é de caráter subjetivo e varia de observador para observador. Diante do exposto, esse tipo de avaliação não pode ser o único empregado para se avaliar técnicas de fusão, sendo necessário também métodos estatísticos. Nesta pesquisa, a avaliação estatística foi baseada nos seguintes indicadores: Média, desvio padrão, IQI (índice de qualidade da imagem) e coeficiente de correlação entre bandas. A média e o desvio padrão referem-se ao brilho e contraste da imagem.

O coeficiente de correlação representa o grau de relação linear existente entre duas variáveis quantitativas (Equação 1).

$$
C C=\frac{\sigma_{\mathrm{xy}}}{\sigma_{\mathrm{x}} \sigma_{\mathrm{y}}}
$$

Em que $\mathrm{CC}=$ coeficiente de correlação

$\sigma_{\mathrm{xy}}=$ covariância entre as imagens $\mathrm{x}$ e y;

$\boldsymbol{\sigma}_{\mathbf{x}}=$ desvio padrão de $\mathrm{x}$;

$\boldsymbol{\sigma}_{\mathbf{y}}=$ desvio padrão de $\mathrm{y}$.
Para medir a qualidade da imagem, foi utilizado o Índice universal de Qualidade de Imagem (IQI) definido matematicamente em Wang e Bovik (2002), Equação 2.

$$
\mathrm{IQI}=\frac{\sigma_{\mathrm{xy}}}{\sigma_{\mathrm{x}} \sigma_{\mathrm{y}}} \cdot \frac{2 \overline{\mathrm{xy}}}{(\mathrm{x})^{2}}+\overline{(\mathrm{y})^{2}} \cdot \frac{2 \sigma_{\mathrm{x}} \sigma_{\mathrm{y}}}{\sigma_{\mathrm{x}}^{2}+\sigma_{\mathrm{y}}^{2}}
$$

Onde x e y são, respectivamente, a banda da imagem multiespectral original e da imagem fusionada. A primeira componente representa o coeficiente de correlação entre x e y (variando de -1 a 1). A segunda componente mede a variação de brilho entre $\mathrm{x}$ e $\mathrm{y}$ (variando entre 0 e 1). A terceira componente mede a similaridade do contraste entre as imagens (variando de 0 a 1) e seu maior valor é obtido quando a variância de $\mathrm{x}$ é igual à variância de $\mathrm{y}$.

\section{Resultados e Discussão}

A etapa dos resultados foi subdividida em duas para facilitar a compreensão das análises qualitativas e quantitativas

\section{Avaliação Qualitativa}

A composição das imagens originais bem como os resultados da fusão das imagens do satélite Landsat8 utilizando o método IHS podem ser visualizados na Figura 4.

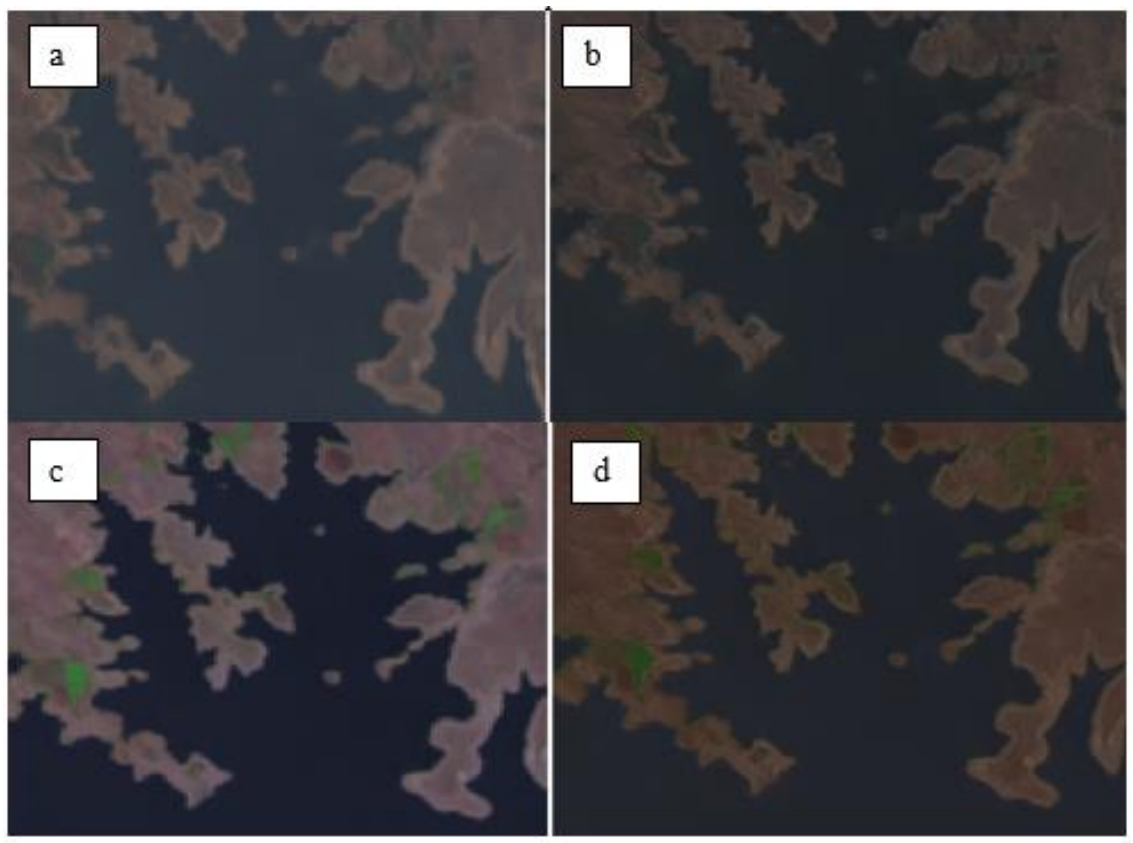

Figura 4 - a) Imagem original composição colorida banda 2 (B), banda $3(G)$, banda $4(R)$; b) Imagem fusionada das bandas 2,3 e 4 com a pancromática; c) Imagem original composição colorida banda 4 (B), banda 5 (G), banda 5 (R); d) Imagem fusionada das bandas 4,5 e 6 com a pancromática. Fonte: os autores (2018) 
$\mathrm{Na}$ análise qualitativa, avaliou-se visualmente a imagem fusionada em comparação com as imagens originais com base em parâmetros ópticos, como padrão geométrico, tamanhos de objetos e cor (Du et al., 2007).

Segundo Fonseca et. al. (2011), nesse tipo de análise há uma dependência principalmente das condições de visualização e da experiência do operador. Analisando a Figura 4, percebemos que as imagens após serem fusionadas apresentam melhores resultados em termos de visualização e definição dos objetos que a compõem. Também podemos perceber que houve uma variação de cores bastante significativa, destacando-se as áreas cobertas com vegetação, tendo suas cores variando do verde brilhante até um verde mais opaco.

Resultados semelhantes a esses podem ser encontrados em (Pushparaj e Hegde, 2017), em que é feita uma análise qualitativa da fusão por diferentes técnicas em imagens quickbird e Landsat8. Um grupo de oito especialistas em sensoriamento remoto (com qualificação de mestrado em sensoriamento remoto e SIG) avaliou a qualidade das imagens com base em melhorias na informação espacial, preservação da informação espectral e distorção espacial e espectral. Neste trabalho, as imagens fusionadas por IHS apresentaram desempenho satisfatório.

\section{Avaliação Quantitativa}

Esta etapa teve como objetivo verificar a fidelidade espectral da imagem fusionada com relação à imagem multiespectral original.

Pushparaj e Hegde (2017) utilizaram catorze índices para avaliar a qualidade espectral e espacial das imagens fusionadas do Landsat8 e Quickbird2 por dez diferentes técnicas de fusão de imagens. Os resultados apontaram que o método IHS foi considerado bom quando comparado a outros métodos.

Nas Tabelas 2 e 3 encontram-se os resultados das médias dos pixels, desvio padrão e variância para a imagem multiespectral original e as imagens fusionas. A média e o desvio padrão estão relacionados com o brilho e contraste da imagem, respectivamente. Quando há uma menor variação na média, o brilho é conservado. Uma menor variação entre os valores de desvio padrão demonstra melhor preservação do contraste da imagem. Analisando as tabelas e comparando os resultados, a imagem fusionada das bandas 2,3 e 4 forneceu bons resultados para o valor da média, desvio padrão e variância, indicando uma conservação do brilho e contraste da imagem não havendo uma alteração significativa quando comparado ao da imagem original. Já na fusão das bandas 4,5 e 6 o resultado não foi o mesmo e houve grandes variações na média, desvio padrão e variância.

Tabela 2 - Média, desvio padrão e variância das imagens multiespectrais originais e da fusionada, bandas 2,3 e 4 .

\begin{tabular}{cccccccccc}
\hline & \multicolumn{3}{c}{ Banda 2 } & \multicolumn{2}{c}{ Banda 3 } & \multicolumn{2}{c}{ Banda 4 } \\
\cline { 2 - 8 } Imagem & Média & $\begin{array}{l}\text { Desvio } \\
\text { padrão }\end{array}$ & Variância & Média & $\begin{array}{c}\text { Desvio } \\
\text { padrão }\end{array}$ & Variância & Média & $\begin{array}{c}\text { Desvio } \\
\text { padrão }\end{array}$ & Variância \\
\hline $\begin{array}{c}\text { Multiespectral } \\
\text { original }\end{array}$ & 7538,2 & 4929,6 & 24300000,0 & 7492,6 & 4960,7 & 24600000,0 & 8350,0 & 5631,6 & 31700000,0 \\
$\begin{array}{c}\text { Imagem } \\
\text { fusionada }\end{array}$ & 7434,2 & 4876,8 & 23800000,0 & 7389,5 & 4907,8 & 24100000,0 & 8233,0 & 5567,26 & 31000000,0 \\
\hline
\end{tabular}

Tabela 3 - Média, desvio padrão e variância das imagens multiespectrais originais e da fusionada, bandas 4,5 e 6 .

\begin{tabular}{cccccccccc}
\hline & \multicolumn{3}{c}{ Banda 4 } & \multicolumn{3}{c}{ Banda 5 } & \multicolumn{2}{c}{ Banda 6 } \\
\cline { 2 - 9 } Imagem & Média & $\begin{array}{c}\text { Desvio } \\
\text { padrão }\end{array}$ & Variância & Média & $\begin{array}{c}\text { Desvio } \\
\text { padrão }\end{array}$ & Variância & Média & $\begin{array}{c}\text { Desvio } \\
\text { padrão }\end{array}$ & Variância \\
\hline $\begin{array}{c}\text { Multiespectral } \\
\text { original } \\
\begin{array}{c}\text { Imagem } \\
\text { fusionada }\end{array}\end{array}$ & 5350,0 & 5632,0 & 31700000,0 & 11349,0 & 7664,0 & 58700000,0 & 14722,0 & 10034,7 & 101000000,0 \\
\hline
\end{tabular}


Através do coeficiente de correlação entre as imagens, foi feita uma análise da transferência da resposta espectral da imagem multiespectral original para as imagens fusionadas (tabela 4).

Tabela 4 - Coeficiente de correlação entre a imagem multiespectral original e a imagem fusionada.

\begin{tabular}{cc}
\hline Coeficiente de correlação \\
\hline Banda 2 & 0,9943 \\
Banda 3 & 0,9948 \\
Banda 4 & 0,9952 \\
Banda 4 & 0,9821 \\
Banda 5 & 0,9867 \\
Banda 6 & 0,9905 \\
\hline
\end{tabular}

Quando o coeficiente de correlação entre as bandas da imagem original e a imagem fusionada apresenta valores elevados próximos de 1 é um fator indicativo de que grande parte das informações na imagem fusionada está coerente com a realidade, ou seja, é uma informação verídica. Dessa forma, é possível fazer análises precisas a partir dessas imagens. Analisando as tabelas acima, percebemos que os resultados foram muito bons para todas as bandas.

Os valores de IQI variam de -1 a 1 e o valor máximo (1) é alcançado quando $\sigma \mathrm{x}=\sigma \mathrm{y}$.

Podemos observar pelos valores de IQI na Tabela 5, que a técnica de fusão aplicada as bandas 2,3 e 4 apresentou melhores resultados em termos de qualidade.

Tabela 5 - Valores de IQI referentes a cada banda da imagem multiespectral original em relação a respectiva banda da imagem fusionada.

\begin{tabular}{ll}
\hline \multicolumn{2}{c}{ IQI } \\
\hline Banda 2 & 0,9940 \\
Banda 3 & 0,9945 \\
Banda 4 & 0,9949 \\
Banda 4 & 0,6829 \\
Banda 5 & 0,6772 \\
Banda 6 & 0,6683 \\
\hline
\end{tabular}

Quanto à distribuição dos níveis de cinza em cada banda, a comparação entre os histogramas imagem original / imagem simulada apresentou resultados interessantes. Observa-se no geral que os histogramas estão coerentes com a imagem original havendo apenas um deslocamento acarretando em mudanças nas médias e desvios padrões, exceção das bandas 5 e 6 (Figura 5). 


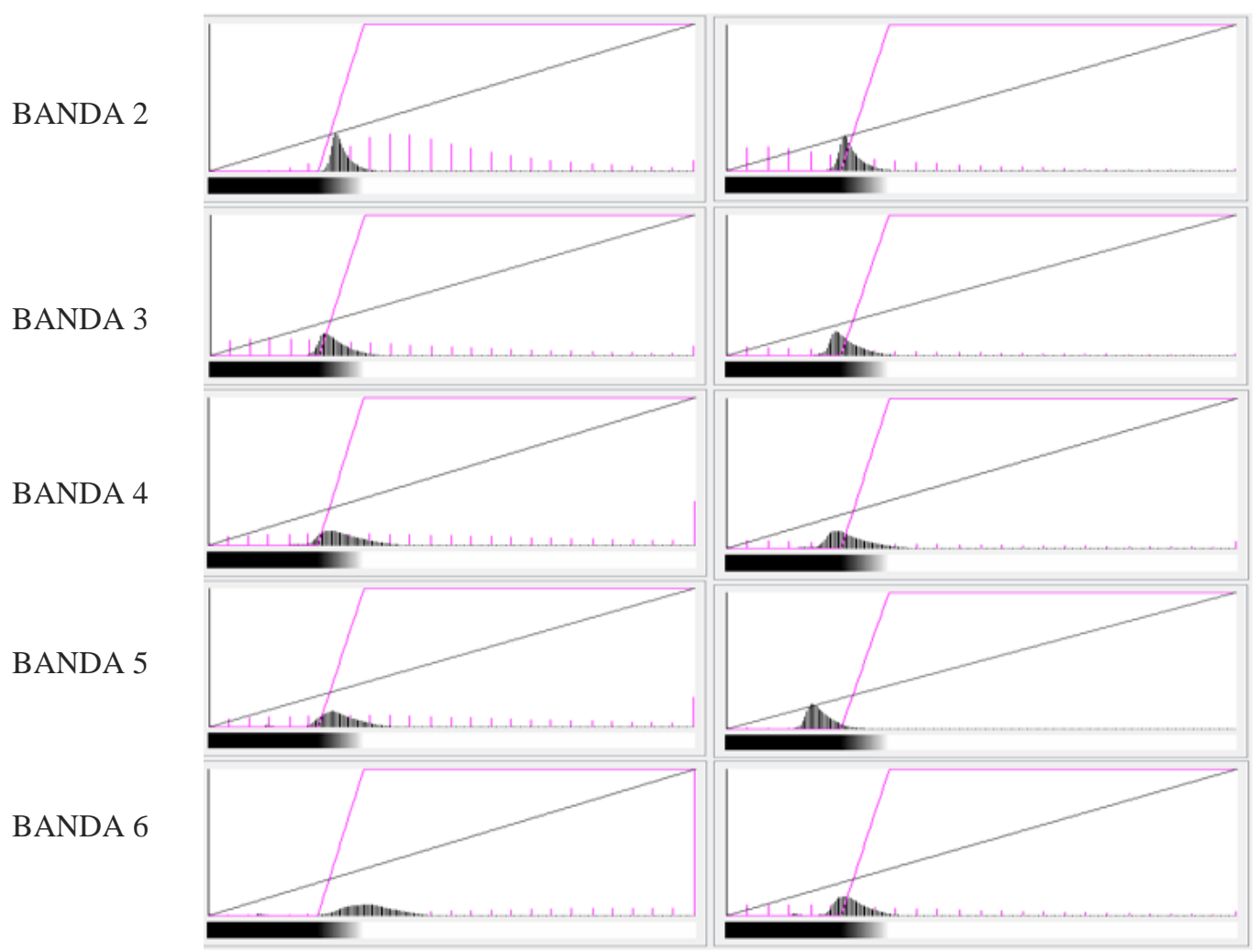

Figura 5 - Histograma das bandas 2, 3,4,5 e 6 antes (esquerda) e após (direita) a fusão. Fonte: os autores (2018).

\section{Conclusões}

Foi apresentado neste trabalho um estudo sobre a técnica de fusão IHS aplicadas em imagens do satélite Landsat 8 . Através da aplicação deste método foi possível perceber uma melhora significativa na qualidade geométrica das imagens multiespectrais de baixa resolução, fazendo com que elas possam ser utilizadas em diversos tipos de projetos.

Além disso, o fato das imagens do Landsat 8 serem disponibilizadas gratuitamente na internet aumenta a viabilidade do uso desta técnica de fusão, pois através dela pode-se obter um produto de boa resolução espectral com maior nível de detalhes e um custo baixo. Imagens resultantes do processo de fusão tem diversas aplicações, dentre elas aplicações ambientais, como cálculos de índices de vegetação, monitoramento de reservatórios de água, dentre outras.

Em contrapartida, apesar de ser uma técnica bastante utilizada, a fusão pelo método IHS possui a limitação de permitir apenas a fusão de três bandas por vez, com a imagem de alta resolução espacial.

\section{Agradecimentos}

Os autores agradecem ao Instituto Nacional de Pesquisas Espaciais (INPE) pela política de distribuição gratuita das imagens do satélite Landsat 8, ao Conselho Nacional de Desenvolvimento Científico e Tecnológico $(\mathrm{CNPq})$ pela bolsa de apoio para o primeiro autor.

\section{Referências}

Du, Q., Gungor, O., Shan, J., 2005 Performance Evaluation for Pan-sharpening Techniques. IEEE Geocience and Remote Sensing Letters 4264-4266.

Fonseca, L., Namikawa, L., Castejon, E., Carvalho, L., Pinho, C., Pagamisse, A., 2011. Image Fusion for Remote Sensing Applications, in: Zheng, Y. (Ed.), Image Fusion and its Applications. InTech, pp. 153178.

Magalhães, I.A.L., Quinto, V.M., Pena, F.E.R., Omena, M.S., Chiba, M.L., Santos, A.R., 2011. Análise comparativa entre Técnicas de fusão de imagens de alta resolução espacial do satélite Quickbird. Caderno de Geociências 8, 82-91.

Meneses, P.R., Almeida, T., 2012. Introdução ao 
Processamento de Imagens de Sensoriamento Remoto. Universidade de Brasília, Brasília.

Pushparaj, J., Hegde, A.V., 2017. Comparison of various pan- sharpening methods using Quickbird-2 and Landsat-8 imagery. Arabian Journal of Geociences 10, 119.

Schneider, M.J., Bellon, O.R.P., Arak, H., 2003. Experimentos em Fusão de Imagens de Alta Resolução. Boletim de Ciências Geodésicas 9, 7588.

Silva, M.M., Candeias, A.L.B., Silva, V.O.F., Carvalho, R.C.O., 2007. Family
Agriculture in Brazilian Semi-Arid, in: Gunkel, G., Sobral, M.C. (Eds.), Reservoir and River Basin Management. Technical University of Berlin, Berlin, pp. 227-238.

Wang, X., Liu, Y., Ling, F., Xu, S., 2017. Fine spatial resolution coastline extraction from Landsat-8 OLI imagery by integrating downscaling and pansharpening. Remote Sensing Letters 9, 314-323.

Wang, Z., Bovik, A.C., 2002. A Universal Image Quality Index. IEEE Signal Processing Letters 9, 81-84. 